\title{
Paracoccidioidomicosis en población pediátrica: revisión de literatura
}

\author{
Paracoccidiodomycosis in pediatric population: literature review \\ Daniel S González-Restrepo,* Iván Benavides,* Lilia Ramírez,* Pío López*,‡ \\ * Facultad de Medicina de la Universidad del Valle. Cali, Colombia. \\ ‡ Presidente de la Sociedad Latinoamericana de Infectología Pediátrica (SLIPE). México.
}

\section{RESUMEN}

La paracocciodiodomicosis es una enfermedad que generalmente se presenta en hombres adultos cuya labor está asociada a actividades agrícolas en América Latina. En dicha población, la micosis se manifiesta de forma crónica; mientras que en la población pediátrica su prevalencia es menor, la afectación pulmonar no es frecuente y se manifiesta de forma aguda/subaguda. A continuación, presentamos el caso de una paciente de siete años de edad a quien se le diagnosticó esta micosis mediante biopsia hepática. Se describen los hallazgos clínicos y paraclínicos y una revisión de la literatura.

Palabras clave: Población pediátrica en paracoccidioidomicosis, paracoccidioidomicosis.

\section{REPORTE DE CASO}

La paracoccidioidomicosis es una enfermedad micótica sistémica causada por Paracoccidioides brasiliensis y Paracoccidioides lutzii. La mayoría de los casos ocurren en hombres adultos que trabajan en el área agrícola. Los reportes de casos en población pediátrica son escasos en la literatura. A continuación, presentamos un caso de paracoccidioidomicosis pediátrica en Colombia.

Paciente femenino de siete años de edad proveniente de Cartago, Valle del Cauca. Presentó síntomas de 10 días de evolución consistentes en palidez, ictericia, elevaciones térmicas cuantificadas a $38^{\circ} \mathrm{C}$ y dos episodios de epistaxis. Como antece-

Financiamiento: Ninguno.

Conflicto de intereses: Ninguno.

Rev Latin Infect Pediatr. 2021; 34 (1): 48-50

\section{ABSTRACT}

Paracoccidioidomycosis is a disease that generally occurs in adult men whose labor is associated with agricultural activities in Latin America. In this population the mycosis manifests in a chronic form. On the other hand, in the pediatric population its prevalence is lower, lung involvement is not common and it is expressed in an acute/subacute form. Next, we present the case of a 7-yearold female patient who was diagnosed with this mycosis by liver biopsy. The clinical and paraclinical findings and a literature review are described.

Keywords: Pediatric population in paracoccidiodomycosis, paracoccidioidomycosis.

dente patológico contaba con diagnóstico de púrpura trombocitopénica idiopática.

En la exploración física de ingreso encontramos múltiples adenopatías móviles no dolorosas a nivel cervical, supraclavicular e inguinal. Entre las pruebas paraclínicas solicitadas en la evaluación, llama la atención hemograma con leucocitosis: $20,880 \times 10^{3}$, neutrofilia: $10,770 \times 10^{3}$, eosinofilia: $2,460 \times 10^{3}$, linfocitos: $5,990 \times 10^{3}$ y monocitos: $1,550 \times 10^{3}$. Además anemia con hemoglobina: $5.3 \mathrm{~g} / \mathrm{dL}$, hematocrito: $19.7 \%$, plaquetas: $68,000 \mathrm{~mm}^{3}$.

Se realizó tomografía axial computarizada (TAC) abdominal, la cual evidenció adenopatías extensas en hilio hepático (Figura 1A), peripancreáticas, mesentéricas y retroperitoneales. TAC de tórax con

Citar como: González-Restrepo DS, Benavides I, Ramírez L, López P. Paracoccidioidomicosis en población pediátrica: revisión de literatura. Rev Latin Infect Pediatr. 2021; 34 (1): 48-50. https://dx.doi.org/10.35366/99828 www.medigraphic.com/infectologiapediatrica 
Rev Latin Infect Pediatr. 2021; 34 (1): 48-50

parénquima pulmonar sin consolidación, pero con presencia de lesiones óseas osteolíticas subcostales, en omóplato y vértebra T12 (Figura 1B).

Se realiza aspiración de médula ósea, cuyo reporte muestra un marcado aumento de eosinófilos, sin presencia de microorganismos ni blastos. Para finalizar, se realizó una biopsia de ganglio linfático hepático, que confirma la presencia de estructuras fúngicas compatibles con paracoccidioidomicosis con extensas áreas de necrosis (Figura 2).

En la actualidad, la paciente se encuentra estable, con adecuada evolución clínica bajo manejo con anfotericina B liposomal.

\section{DISCUSIÓN}

La paracoccidioidomicosis es una micosis sistémica, profunda y progresiva causada por un hongo termodimórfico, Paracoccidioides brasiliensis, el cual está formado por cuatro especies filogenéticas diferentes: PS1, PS2, PS3 y PS4 y en menor medida por una especie recientemente descrita, Paracoccidioides lutzii, ubicado en el centro de Brasil. ${ }^{1}$ Ésta es una enfermedad endémica en áreas húmedas tropicales y subtropicales de América Latina ricas en materia orgánica, arroyos, ríos y vegetación. Es común en Brasil, donde se detecta el $80 \%$ de todos los pacientes y donde es una de las principales causas de mortalidad. ${ }^{2,3}$

La mayoría de los pacientes tienen 30 años de edad o más. Suele afectar a hombres que se desempeñan en tareas agrícolas. La micosis es rara en niños $(<2 \%)$ y adolescentes $(<9 \%)$. Varias series con más de 5,000 pacientes destacaron que la relación entre hombres y mujeres era de 11:1.1, respectivamente. Una explicación a esto es la acción de la hormona femenina 17-B-estradiol, la cual tiene un efecto inhibidor sobre la fase de levadura del hongo. La ausencia de estos factores en los niños explica por qué la presentación de la enfermedad es similar en ambos sexos. ${ }^{4}$

La micosis puede presentarse de forma aguda/ subaguda, la cual es común en niños y adolescentes, (como es el caso de nuestro paciente) o de forma crónica, que corresponde a la mayoría de presentaciones clínicas (90\%) y se diagnostica en adultos.

La forma aguda/subaguda se caracteriza por linfadenopatía difusa, hepatoesplenomegalia, diarrea e ictericia. Rara vez se observa afectación pulmonar. A nivel paraclínico se puede encontrar leucocitosis con eosinofilia, anemia, hipergammaglobulinemia e hipoalbuminemia. ${ }^{2}$ Nuestra paciente presentó hallazgos clínicos y paraclínicos publicados en la literatura.

El diagnóstico se basa en la identificación directa del hongo con el microscopio en su fase de levadura, donde se observará la gemación múltiple, la cual da una imagen característica de rueda de timón. El cultivo, la evaluación histopatológica mediante tinciones especiales de plata con metenamina ácido peryódico de Schiff (PAS) o Grocott-Gomori y la reacción en

Figura 1:

A) Múltiples adenopatías a nivel hepático. B) Lesiones osteolíticas a nivel de costilla y omóplato.
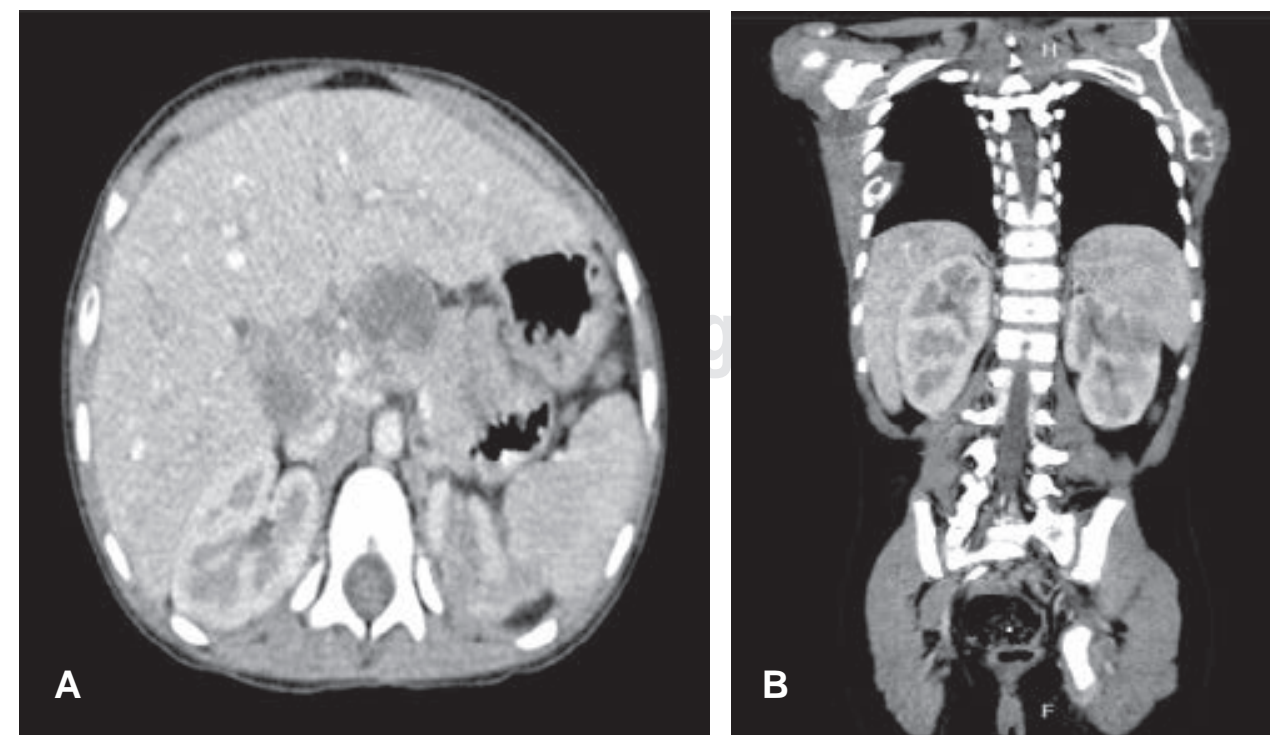


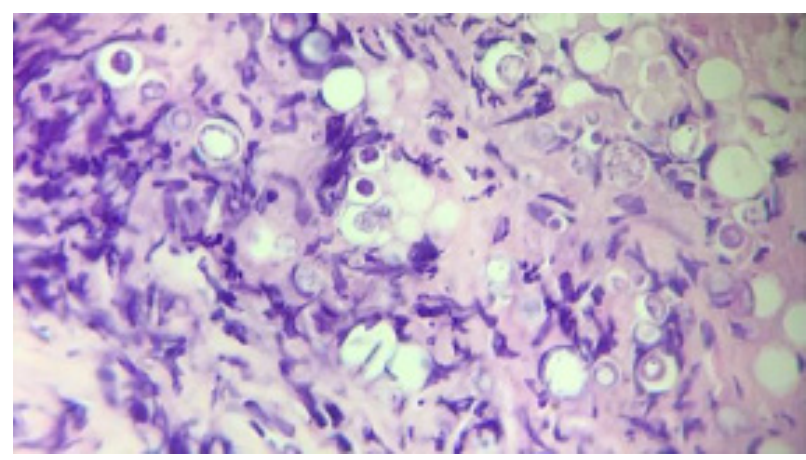

Figura 2: Parénquima hepático con infiltrado inflamatorio crónico portal con componente de eosinofílico, histiocitos epitelioides y abundantes microorganismos encapsulados, esféricos eosinofílicos. Estructuras micóticas positivas para ácido peryódico de Schiff (PAS, por sus siglas en inglés) y Grocott-Gomori.

cadena de la polimerasa son herramientas que también han demostrado su utilidad. ${ }^{1-5}$

En los niños, la duración total precisa de la terapia varía según la gravedad de la enfermedad y de la respuesta clínica del paciente al tratamiento.

Muchos expertos prefieren la anfotericina B para el tratamiento inicial de la paracoccidioidomicosis grave. La terapia oral con itraconazol es el tratamiento de elección para infecciones menos severas o localizadas, y para completar el tratamiento cuando se emplea anfotericina B inicialmente. ${ }^{6-8}$

Por otro lado, la paracoccidioidomicosis es la única enfermedad fúngica que puede tratarse con éxito con sulfamidas. ${ }^{5-9}$ El trimetoprim-sulfametoxazol intravenoso es una alternativa, puede administrarse inicialmente por vía intravenosa y después hacer un cambio a vía oral cuando se haya observado una mejoría clínica, generalmente después de tres a seis semanas. La duración del tratamiento total suele ser de seis a 12 meses. ${ }^{10}$

\section{REFERENCIAS}

1. Benett JE, Dolin R, Blaser MJ. Mandell, Douglas y Bennett. Enfermedades infecciosas: principios y práctica. Capítulo 269. 8a ed. Barcelona: Elsevier; 2015. pp. 3166-3174.

2. Martinez R. New trends in paracoccidioidomycosis epidemiology. J Fungi (Basel). 2017; 3 (1): 1.

3. Martinez R. Epidemiology of paracoccidioidomycosis. Rev Inst Med Trop Sao Paulo. 2015; 57 (Suppl 19): 11-20.

4. Ryan ET, Hill DR, Solomon T, Aronson N, Endy TP. Hunter's tropical medicine and emerging infectious diseases. Chapter 80. 10th edition. London: Elsevier; 2020. p. 674.

5. Choque Barrera M, Suárez Barrientos EL, Calderón López ME. Paracoccidioidomicosis, a propósito de un caso. Gac Med Bol. 2015; 38 (1): 30-33.

6. Cherry J, Demmler-Harrison GJ, Kaplan SL, Steinbach WJ, Hotez P. Feigin and cherry's textbook of pediatric infectious diseases. 8th ed. Philadelphia, PA: Elsevier; 2019.

7. American Academy of Pediatrics. Paracoccidioidomycosis. In: Kimberlin DW, Brady MT, Jackson MA, Long SS, eds. Red Book: 2018 Report of the Committee on Infectious Diseases. 30th ed. Elk Grove Village, IL: American Academy of Pediatrics; 2018. p. 590.

8. de Arruda JAA, Schuch LF, Abreu LG, Silva LVO, Mosconi C, Monteiro JLGC et al. A multicentre study of oral paracoccidioidomycosis: analysis of 320 cases and literature review. Oral Dis. 2018; 24 (8): 1492-1502. doi: 10.1111/ odi.12925.

9. Cole DC, Govender NP, Chakrabarti A, Sacarlal J, Denning DW. Improvement of fungal disease identification and management: combined health systems and public health approaches. Lancet Infect Dis. 2017; 17 (12): e412-e419.

10. Trindade AH, Meira HC, Pereira IF, de Lacerda JCT, de Mesquita RA, Santos VR. Oral paracoccidioidomycosis: retrospective analysis of 55 Brazilian patients. Mycoses. 2017; 60 (8): 521-525.

Correspondencia:

Dr. Pío López

E-mail: pio.lopez@ceiponline.org 